\title{
Anti-Phosphatidylserine Antibodies in Acute Myocardial Infarction
}

\author{
1,2 Abdolreza Sotoodeh Jahromi, ${ }^{1}$ Mohammad Shojaei, \\ ${ }^{3}$ Mohammad Reza Farjam and ${ }^{4}$ Abdolhossien Madani \\ ${ }^{1}$ Atherosclerosis Research Center, \\ ${ }^{2}$ Department of Immunology, \\ ${ }^{3}$ Research Center for Social Determinants of Health, \\ Faculty of Medicine, Jahrom University of Medical Science, Jahrom, Iran \\ ${ }^{4}$ Center for Determinants in Health Promotion, \\ Faculty of health, Hormozgan University of Medical Science, Bandarabbas, Iran
}

Received 2013-09-15, Revised 2013-09-19; Accepted 2013-09-21

\begin{abstract}
Acute Myocardial Infarction (AMI) is the combined result of environmental factors and personal predispositions. Many factors play a role in AMI including anti-Phospholipid (aPL) antibodies, that may act in the induction of immunological response leading to the development of AMI. AntiPhosphatidylserine (PS) antibody is detected in various diseases like rheumatoid arthritis, systemic lupus erythematosus and anti-phospholipid antibody syndrome. The study of anti-PS antibody in AMI might shed light on etiologic mechanisms in the pathogenesis of acute coronary syndromes. This study was conducted to evaluate whether prevalence of anti-PS antibodies, in patients who had AMI and to analyze their relationship with traditional cardiovascular risk factors. The prevalence of anti-PS IgG and IgM in a well characterized group of patients with AMI as a case group and in age and sex matched healthy subjects as control group. Sera from two groups were tested to evaluate the presence of IgG and IgM isotypes to anti-PS by ELISA method. The frequencies of positive test for anti-PS IgG were 26.70 and $8.90 \%$ among patients and controls respectively with significant difference $(p=0.003)$. The anti-PS IgM frequencies were 12.20 and $1.10 \%$ in patients and the controls, with significant difference $(p=0.005)$. The findings of this study suggest that anti-PS antibodies seemed to play a role in AMI, independent risk factors for AMI, which may represent a link between autoimmunity and atherosclerosis in patients with AMI. Further studies with bigger sample size including patients with AMI and healthy people are recommended to explore the exact role of anti-PS antibodies in AMI.
\end{abstract}

Keywords: Anti-Phosphatidylserine (PS) Antibodies, Acute Myocardial Infarction (AMI), AntiPhospholipid (aPL) Antibodies, Cardiovascular Ischemia

\section{INTRODUCTION}

Cardiovascular Disease (CVD) is one of the important causes of deaths despite developments in the medical interventions. Among these, the ischemic heart diseases, Acute Myocardial Infarction (AMI) in particular, is one of the most alarming values (Upaganlawar et al., 2011).

Myocardial Infarction (MI) is the collective result of environmental factors, personal predispositions and genetics (Shojaie et al., 2013). Factors such as low serum adiponectin (Shojaie et al., 2009a) and low Jahrom University of Medical Science, Jahrom, Iran 
annexin V levels (Shojaie et al., 2009b) and infectious diseases such as Mycoplasma pneumonia (Pourahmad et al., 2009) are a part of involving factors in AMI. It has been demonstrated that the immunopathological such as anti-beta2 glycoprotein-I antibody (Shojaie and Jahromi, 2011) and Inflammatory processes (Jahromi et al., 2010a) have important roles in the initiation and development of AMI.

Antiphospholipid antibodies (aPL) in addition to habitual abortion and pregnancy loss (Jahromi et al., 2010b) are associated with CVD (Shojaie and Jahromi, 2011; Jafarzadeh et al., 2011). However their pathogenic mechanisms are still matter of investigation.

Besides the Classical Lupus Anticoagulant (LAC) and Anti-Cardiolipin Antibodies (ACA), other antiPhospholipid Antibodies (aPL) were shown to target anionic phospholipids and other plasma proteins, including phosphatidylethanolamine, protein $\mathrm{C}$, protein S, B2-Glycoprotein I (beta2-gpI) and annexin $\mathrm{V}$ (Tincani et al., 2010).

The results of some studies also demonstrated a realationship between anti-PL antibody and IHD (Shojaie and Jahromi, 2011; Jafarzadeh et al., 2011; Hisham et al., 2007). It should be noted that genetic factors as well as other traditional risk factors such as smoking, hypercholesterolemia, diabetes mellitus and hypertension may contribute to IHD development and these parameters differ among various population. Even though there are a few studies on the association of some autoantibodies with AMI, more epidemiological data are needed to confirm their significance as independent risk factors in CVD. Furthermore, the data on the correlation of autoantibodies with traditional risk factors of AMI is scarce. Hence, this case control study was conducted to evaluate the serum levels of Anti-Phosphatidylserine (PS) antibodies (IgG and IgM) in Iranian patients with AMI and also to explain their association with some traditional risk factors of this disease.

\section{MATERIALS AND METHODS}

\subsection{Subjects}

A total of 90 consecutive patients (aged 41-67 years) with AMI including 67 men and 23 women who were admitted to Peymanieh Hospital of Jahrom (a southwest city of Iran), were enrolled to this casecontrol study.

AMI was diagnosed by the presence of two of the following criteria: (i) prolonged chest pain compatible with AMI, (ii) typical ECG changes, (iii) rising of cardiac enzymes such as creatine kinase and lactate dehydrogenase (Kushner et al., 2009).

Exclusion criteria were valvular heart disease, surgery, trauma during the prior month, cardiomyopathy, liver disease, renal failure, arthritis, malignant diseases, other inflammatory diseases (such as rheumatoid arthritis and systemic lupus erythematosus) and oral anticoagulant therapy. In patients with AMI, the serum concentration of Anti-PS antibodies was measured during 3-5 days after admission.

An age, sex and other CAD risk factors such as Hypertension (HTN), Diabetes Mellitus (DM) and Hyper Lipidemia (HLP) matched group $(n=90)$ with similar geographic and socioeconomic backgrounds without any ischemic heart disease were used as a control group. All control subjects were basically healthy, with no acute or chronic illnesses and did not use any drugs. The healthy control group was recruited from blood donors attending Jahrom Blood Transfusion Center.

The study protocol was approved by research ethics committee of Jahrom University of Medical Sciences and informed consents were obtained from all participants before enrollment. The patient or his/her legal representative provided written informed consent.

Historical, demographic and clinical data were obtained through a review of medical records and interviews with patients and their families. The risk factors for myocardial infarction were as following.

(a) age, sex; (b) history of hypertension (diagnosis confirmed when the systolic or diastolic pressures were $>160$ or $95 \mathrm{~mm} \mathrm{Hg}$, respectively, or when the patient was using antihypertensive medication) 12; (c) smoking, according to the criteria of the British Council for Medical Research; (d) history of heart disease (atrial fibrillation or coronary heart disease, defined as previous myocardial infarction, angina, or revascularization procedure); (e) history of diabetes mellitus, according to the medical history or the use of insulin or an oral anti-diabetes drug; (f) hypercholesterolemia, based on total cholesterol $>200 \mathrm{mg} \mathrm{dL}^{-1}$, LDL-cholesterol $>130$ $\mathrm{mg} \mathrm{dL}^{-1}$, or total cholesterol/HDL-cholesterol ratio $>5$ (Donahue et al., 1988). 
Blood samples (5 cc) were obtained by venipuncture from the patients immediately after admission before starting any IV medications by skilled personnel. Blood samples were centrifuged and frozen within, at most, $2 \mathrm{~h}$ after collection and stored at $-20^{\circ} \mathrm{C}$ until laboratory testing.

Enzyme-immunoassay kits (Orgentic Diagnostika from Mainz, Germany) were used to detect IgG or IgM anti-PS by indirect enzyme linked immunosorbent assay (ELISA) method. The $\operatorname{IgG}$ and IgM isotypes results were assessed in IgG Phospholipid (GPL) and IgM phospholipid (MPL) units, with one unit equal to $1 \mu \mathrm{g} \mathrm{mL}^{-1}$ of $\operatorname{IgG}$ or IgM. They were considered positive if the titer was $>10$ GPL units and $>10$ MPL units for both isotypes, as recommended by the ELISA kits manufacturer.

Statistical analyses were performed by SPSS (version 17; SPSS, Inc., Chicago, IL). Data were expressed as mean \pm SD. Continuous variables with little-to-mild skewness were summarized as mean \pm SD and compared using Student's t-test.

\section{RESULTS}

The demographic and clinical characteristics of the study groups, as well as laboratory findings are shown in Table 1.

In the patient group, 16 cases $(18.80 \%)$ had Non-St Elevation MI (NSTEMI) and $74(82.20 \%)$ had ST Elevation MI (STEMI).

There was no significant difference between the two groups regarding the following variables: age, sex, HTN, DM, LDL, HDL, total cholesterol and TG.
We examined the association between positive anti-PS IgG and IgM tests and selected cardiovascular risk factors.

There was a significant association between positive anti-PS IgG and IgM tests with Type hypertension. But there was not found any significant association between positive anti-PS IgG and IgM test with Type $1 \mathrm{DM}$, Type 2 DM, age, sex, LDL, HDL, TG, total cholesterol and adjusted smoking.

There was not found significant difference between positive anti-PS IgG tests in patients with STEMI and those with NSTEMI $(73.30$ Vs $25.90 \%),(p=0.186)$ and also there was not found significant difference between positive anti-PS IgM tests in patients with STEMI and those with NSTEMI (74.10 Vs 26.70\%), $(p=0.324)$.

\section{DISCUSSION}

The results of the present study showed that the prevalence of positive anti-PS IgG and also IgM in patients with AMI were significantly higher than that observed in healthy control group. These associations were independent of traditional risk factors. It was found that the high prevalence of anti-PS antibodies in patients with AMI Vs healthy control group in parallel with previous studies (Jafarzadeh et al., 2011; Hisham et al., 2007) consistent with current concepts on the immune pathogenesis of atherosclerosis. However, the precise mechanisms of involvement of anti-PS antibodies in the pathogenesis of AMI remain to be determined.

An association between CAD and AMI with Other aPLs have been reported in previous studies (Shojaie and Jahromi, 2011; Jafarzadeh et al., 2011; Greco et al., 2010).

Table 1. Demographic, clinical characteristics and laboratory finding for the case and the control groups

\begin{tabular}{llll}
\hline Variables & Patients group & Control group & P-value \\
\hline Age (year) & $9.63 \pm 62.66$ & $10.63 \pm 60.53$ & 0.609 \\
Male (\%) numbers & $67(74.40 \%)$ & $68(75.60 \%)$ & 0.863 \\
High blood pressure (\%) numbers & $39(43.33 \%)$ & $28(31.10 \%)$ & 0.079 \\
Smoker (\%) numbers & $40(44.40 \%)$ & $32(35.60 \%)$ & 0.287 \\
IDDM * (\%) numbers & $24(26.70 \%)$ & $28(31.10 \%)$ & 0.622 \\
Total cholesterol * Mg/dL & $40.30 \pm 176.13$ & $43.20 \pm 168.80$ & 0.104 \\
HDL-C * Mg/dL & $9.11 \pm 44.20$ & $10.29 \pm 42.83$ & 0.328 \\
LDL-C * Mg/dL & $36.32 \pm 118.05$ & $30.64 \pm 98.22$ & 0.093 \\
TG $*$ Mg/dL & $72.18 \pm 126.53$ & $41.52 \pm 114.84$ & 0.104 \\
FBS $*$ Mg/dL & $54.48 \pm 134.20$ & $53.91 \pm 127.08$ & 0.495 \\
Positive Anti-PS IgG (\%) numbers & $24(26.70 \%)$ & $8(8.90 \%)$ & 0.003 \\
Positive Anti-PS IgM (\%) numbers & $11(12.20 \%)$ & $1(1.10 \%)$ & 0.005 \\
\hline
\end{tabular}

*IDDM $=$ Insuline Dependent Diabetes Mellitus, NIDDM = Non-Insuline Dependent Diabetes Mellitus, HDL-C = High Density Lipoprotein-Cholesterol, LDL-C = Low Density Lipoprotein-Cholesterol, FBS = Fasting Blood Sugar, Anti-PS = Anti-Phatidylserine 
There was not found correlation between anti-PS IgG and also IgM and standard cardiovascular risk factors such as smoking, hypertension and diabetes, as seen about other apl antibodies in a previous study (Shojaie and Jahromi, 2011).

In this study there was a significant association between anti-PS IgG with HTN in the case and the control groups. The same results were found about antiPS IgG in AMI in other studies (Hisham et al., 2007). These results suggest that anti-PS IgG are independent of these variables.

As there were not significant differences between positive anti-PS antibodies test in patients with STEMI and those with NSTEMI, it can be concluded that anti-PS IgG and IgM do not participate in kind of AMI (STEMI vs NSTEMI). But, unfortunately, there were not found any data indicating association of anti-PS IgG and IgM with these parameters to compare the results.

\section{CONCLUSION}

These results show an association between AMI and high prevalence of positive tests for anti-PS IgG and $\operatorname{IgM}$. The results of this study in parallel with the data from other studies (Shojaie and Jahromi, 2011; Jafarzadeh et al., 2011; Greco et al., 2009; Urbanus et al., 2009; Hisham et al., 2007) support the potential important role of aPL antibodies in AMI.

Further studies with larger sample size of patients and healthy subjects are recommended to explore the precise role of anti-PS IgG and IgM in AMI.

\section{ACKNOWLEDGEMENT}

This study was completely financed by Jahrom University of Medical Sciences. The authors are grateful to the patients and the control individuals who accepted to enter this study.

\section{REFERENCES}

Kushner, F.G., M. Hand, S.C. Smith Jr, S.B. King and J.L. Anderson et al., 2009. Focused updates: ACC/AHA guidelines for the management of patients with ST-elevation myocardial infarction (updating the 2004 guideline and 2007 focused update) and ACC/AHA/SCAI guidelines on percutaneous coronary intervention (updating the 2005 guideline and 2007 focused update) a report of the American College of Cardiology Foundation/American Heart Association Task force on practice guidelines. J. Am. Coll Cardiol., 54: 2205-2241. PMID: 19942100
Donahue, R.P., R.D. Abbott, D.M. Reed and K. Yano, 1988. Physical activity and coronary heart disease in middle-aged and elderly men: The honolulu heart program. Am. J. Publ. Health, 78: 683-685. DOI: 10.2105/AJPH.78.6.683

Greco, T.P., A.M. Conti-Kelly, J.R. Anthony, T. Greco and R. Doyle 2010. Oxidized-LDL/beta(2)glycoprotein I complexes are associated with disease severity and increased risk for adverse outcomes in patients with acute coronary syndromes. Am. J. Clin. Pathol., 133: 737-743. DOI: 10.1309/AJCP88WVRDRDFBAS

Greco, T.P., A.M. Conti-Kelly, J.R. Greco, R. Doyle and E. Matsuura, 2009. Newer antiphospholipid antibodies predict adverse outcomes in patients with acute coronary syndrome. Am. J. Clin. Pathol., 132: 613-620. PMID:19762540

Hisham, Y., M Ali and Z.A. Abdullah, 2007. Antiphosphatidyl serine autoantibodies and premature coronary events. Sultan Qaboos Univ. Med. J., 7: 227-232. PMCID: PMC3074886

Jafarzadeh, A., M. Poorgholami, M. Nemati and M. Rezayati, 2011. High serum levels of rheumatoid factor and anti-phosphatidylserine antibody in patients with ischemic heart disease. Iran. J. Immunol., 8: 34-44. PMID: 21427494

Jahromi, A.S., M. Shojaie and A. Madani, 2010a. Cardiotrophin-1 in patients with acute myocardial infarction. Am. J. Applied Sci., 7: 1190-1194. DOI: 10.3844/ajassp.2010.1190.1194

Jahromi, A.S., M.R. Farjam and F. Mogharrab, 2010b. Anti $\beta 2$-glycoprotein-I antibodies in women with recurrent spontaneous abortion. Am. J. Biochem. Biotechnol., 6: 264-267. DOI: 10.3844/ajbbsp.2010.264.267

Pourahmad, M., S.A. Jahromy and M. Shojaei, 2009. Association of Mycoplasma pneumonia infection with myocardial infarction. Am. J. Immunol., 5: 8488. DOI: 10.3844 /ajisp.2009.84.88

Shojaie, M. and A.S. Jahromi, 2011. Anti- $\beta 2$ glycoprotein-i antibody in acute myocardial infarction. Am. J. Applied Sci., 8: 758-761. DOI: 10.3844/ajassp.2011.758.761

Shojaie, M., A. Sotoodah and G. Shafaie, 2009a. Is adiponectin associated with acute myocardial infarction in Iranian non obese patients. Lipids Health Dis., 28: 17-17. DOI: 10.1186/1476-511X-817 
Shojaie, M., A. Sotoodah, S. Roozmeh, E. Kholoosi and S. Dana, 2009b. Annexin V and anti-annexin V antibodies: Two interesting aspects in acute myocardial infarction. Thromb. J. DOI: 10.1186/1477-9560-7-13

Shojaie, M., M.Y. Karami, M.K. Jahromi, A.S. Jahromi and A. Makarem, 2013. Different clinical and angiographic finding in a set of Iranian identical twin: A case report. OnLine J. Biol. Sci., 13: 95-98. DOI : 10.3844/ojbsci.2013.95.98

Tincani, A., C. Casu, S. Cartella, T. Ziglioli and R. Cattaneo, 2010. Antiphospholipid antibody: Laboratory, pathogenesis and clinical manifestations. Reumatismo, 62: 65-75. PMID: 20390120
Upaganlawar, A., H. Gandhi and R. Balaraman, 2011. Isoproterenol induced myocardial infarction: Protective role of natural products. Am. J. Pharmacol. Toxicol., 6: 1-17. DOI: 10.3923/jpt.2011.1.17

Urbanus, R.T., B. Siegerink, M. Roest and F.R. Rosendaal et al., 2009. Antiphospholipid antibodies and risk of myocardial infarction and ischaemic stroke in young women in the RATIO study: A casecontrol study. Lancet Neurol., 8: 998-1005. DOI: 10.1016/S1474-4422(09)70239-X 